\title{
Mudanças climáticas e distribuição social da percepção de risco no Brasil*
}

Marcel Bursztyn

\& Flávio Eiró**

Resumo: Este artigo tem por objetivos testar a aplicabilidade da teoria da sociedade de risco de Ulrich Beck para a sociedade brasileira contemporânea e analisar a distribuição social da percepção de risco associada às mudanças climáticas e ao aquecimento global. Para tanto, é feita uma revisão de teorias de risco, além de uma discussão crítica de sua aplicação ao caso das mudanças climáticas. Em seguida são apresentados resultados de pesquisa, verificando o efeito de diferentes variáveis sociodemográficas na percepção de risco. Os dados advêm de pesquisa de opinião pública em território nacional, com amostra estratificada por conglomerados. O principal resultado alcançado diz respeito à homogeneidade da percepção de risco, por meio de diferentes categorias sociais ou contextos geográficos. As únicas categorias que apresentaram influências significativas na avaliação da percepção de risco foram renda familiar e escolaridade, ambas com relação positiva.

Palavras-chave: mudanças climáticas, aquecimento global, risco, percepção, construtivismo.

\section{Introdução}

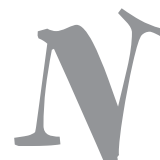

o início da década de 1990 o sociólogo alemão Ulrich Beck (2010) ganhou notoriedade com sua obra $A$ sociedade de risco, onde analisa o mundo ocidental à luz dos riscos ambientais modernos. Beck defende que essas sociedades experimentam mudanças radicais no que tange aos riscos ambientais, cujo notório crescimento é acompanhado de um aumento na percepção dos mesmos por parte da sociedade em geral que, como resultado, altera suas crenças, seus interesses e comportamentos. Aceitas essas premissas para a sociedade ocidental de forma geral, a aplicação das mesmas e, portanto, da teoria da sociedade de risco, em menor escala, pode ajudar na compreensão do fenômeno social estudado por Beck em suas diversas nuanças, com suas particularidades e exceções.

É neste contexto que se insere este trabalho, que tem por objetivos: testar a aplicabilidade da teoria da sociedade de risco de Beck para a sociedade brasileira contemporânea e analisar a distribuição social da percepção de risco associada às mudanças climáticas e ao aquecimento global. Para tanto, na primeira parte, é feita uma revisão crítica das teorias de risco e da corrente construtivista da sociologia ambiental de forma mais ampla. Discute-se a atualidade da teoria de Beck, em especial para o estudo de uma questão específica - o aquecimento global e as mudanças

\author{
* Este artigo \\ teve como base \\ a pesquisa que \\ foi tratada na \\ dissertação de \\ mestrado (Eiró, \\ 2012), na qual \\ os autores foram \\ orientador e \\ orientando. \\ Agradecemos a \\ Fabrízio Machado \\ e Mara do Couto \\ Fernandes \\ pelo convite à \\ participação da \\ pesquisa que deu \\ origem a esse artigo, \\ e a Laura Duarte, \\ Diego Lindoso e \\ Vincent Nédélec \\ por comentários em \\ versões anteriores do \\ trabalho.
}

\footnotetext{
** Marcel Bursztyn

é doutor em

desenvolvimento econômico e social pela Université de Paris 1, professor do Centro de Desenvolvimento Sustentável da Universidade de Brasília<marcel@ unb.br>

Flávio Eiró é doutorando em sociologia na École des Hautes Études en Sciences Sociales de Paris (Centre Maurice Halbwachs), em cotutela com o Centro de Desenvolvimento Sustentável da Universidade de Brasília<flavioeiro@ gmail.com $>$.
} 
1. Em trabalho semelhante em escala regional para o sertão do São Francisco, Bahia, uma discussão complementar foi desenvolvida com a participação de um dos autores (Eiró \& Lindoso, 2014).

2. Posteriormente, Beck publicou artigo dedicado ao tema: "Climate for change, or how to create a green modernity?" (Theory, Culture \& Society, v. 27, n. 2-3, p. 254-266, 2010). Este trabalho, porém, não analisa as mudanças climáticas a partir da ótica da sociedade de risco e não tem por objetivo uma produção de teoria sociológica acerca do tema, trata-se de uma discussão política. O mesmo vale para Anthony Giddens, em seu trabalho $A$ política da mudança climática (Rio de Janeiro: Zahar, 2010). climáticas - e onde estão suas limitações. Em seguida são apresentados resultados de uma pesquisa realizada em território nacional, com amostra estratificada por conglomerados, que teve por objetivo compreender a percepção da sociedade brasileira a respeito das mudanças climáticas.

Ao explorar o significado do fenômeno para diferentes indivíduos e grupos da sociedade brasileira, a partir da verificação do efeito de diferentes variáveis sociodemográficas na percepção de risco, conclui-se que a preocupação acerca do tema é marcada por uma homogeneidade de representações que transpassam diferenças demográficas e geográficas. Apenas duas variáveis apresentaram influência significativa na percepção de risco, com um claro efeito estruturante: renda familiar e escolaridade, ambas com relação positiva. De forma geral, os dados indicam que a grande maioria dos entrevistados acredita que os efeitos do aquecimento global já começaram a aparecer ou aparecerão nos próximos anos, e que suas vidas serão afetadas diretamente por eles. Ademais, esse risco diz respeito a todos os aspectos de suas vidas, mas de forma excepcional - e consensual - aspectos físicos.

\section{Discussão teórica ${ }^{1}$}

A teoria da sociedade de risco é uma das poucas macroteorias sociais a dar enfoque à interação sociedade-natureza. Em sua obra Sociedade de risco: rumo a uma outra modernidade, Ulrich Beck (2010) formula as bases de sua teoria. Mais tarde, ela foi ampliada em Ecological politics in an age of risk (Beck, 1995) e em World risk society (Beck, 1999). Este trabalho, entretanto, concentra sua análise na primeira obra, por entender que ali se encontra a base teórica necessária para uma análise da construção de risco das mudanças climáticas. Vale assinalar que a teoria da sociedade de risco não aborda diretamente o tema da mudança do clima², já que no momento de sua publicação o tema ainda não era tão amplamente debatido como atualmente (Lever-Tracy, 2008).

Os riscos analisados por Beck dizem respeito ao novo estágio, altamente industrializado, da sociedade moderna do fim do século XX. Apesar de já se entender há algum tempo que o aquecimento global está relacionado com ações acumuladas desde os primórdios do industrialismo, e é causado principalmente por tecnologias industriais básicas (IPCC, 2001; 2007), o risco a ele associado enquadra-se na teoria da sociedade de risco. É preciso ressaltar que Beck admite, em sua obra, estar mais interessado em percepções culturais e definições de risco, e não em sua realidade, partindo de uma perspectiva do construtivismo ambiental, que encara os problemas ambientais como produtos de construção social; ou seja, que foram negociados, definidos e legitimados na esfera social (Lidskog, 2001; Olivieri, 2009; Smith, 2001). Segundo Beck, 
se as pessoas vivenciam os riscos como algo real, eles são reais. Se eles são, contudo, reais nesse sentido, eles reviram a estrutura de competências sociais, políticas e econômicas (Beck, 2010: 95).

Outras teorias sociais fazem eco ao trabalho de Beck. A principal talvez esteja nos trabalhos de Mary Douglas e o que ela denominou a teoria cultural do risco (Douglas, 1994). Como Beck, Douglas analisa o desenvolvimento de significados culturais de risco como marca distintiva da modernidade (Wilkinson, 2001). Também Moscovici (2003), em sua teoria das representações sociais, defende que qualquer realidade externa ao ser humano só tem importância na medida em que é representada socialmente pelos indivíduos. A partir da interação entre estes indivíduos, são criadas as representações sociais que, ao mesmo tempo, possibilitam a comunicação e dinamizam transformações na sociedade (Moscovici, 2003; Joffe, 2003; Sancovschi, 2007; Mora, 2002). Finalmente, Jürgen Habermas (1984) enfatiza o caráter ativo dessa construção social em sua teoria da ação comunicativa. O foco de Habermas está na interação e comunicação entre os indivíduos, no que diz respeito ao mundo objetivo (material), social (normas e instituições) e subjetivo (sentimentos) para a construção de uma ação conjunta.

Alguns teóricos trabalharam o outro lado desta organização social: a inação. Do ponto de vista individual, Christine Bérubé (2010) levanta diversas teorias psicológicas que explicam a falta de engajamento no combate ao aquecimento global:

1. incompreensão do fenômeno, em suas causas e efeitos e, por isso, não saber como agir;

2. ausência de impactos perceptíveis, que levam as pessoas a não se preocuparem tanto - também um dos principais pontos na obra de Beck (2010);

3. ação gota d'água: a ideia de que sua contribuição é tão pequena que não faz diferença ${ }^{3}$;

4. saturação de sacrifícios, ou o senso de injustiça de que outras pessoas não farão o mesmo esforço;

5. o problema se resolverá por si, seja pelo desenvolvimento tecnológico - base da teoria da modernização ecológica -, seja por uma força superior (religiosa); e, por fim,

6. a desconfiança no próprio fenômeno, que pode ser uma criação da mídia, uma conspiração, ou um erro científico.

Tais explicações deixam claro que dimensões cognitivas e comportamentais nem sempre estão coerentemente relacionadas. As razões desta incoerência, porém, estão além da esfera psicológica.

3. Aqui é

interessante o paralelo com a teoria dos free riders, de Mancur Olson (1999), que usa esse conceito para explicar o comportamento do "caroneiro", que não contribui com um esforço comum, mas dele não pode ser excluído (Fonseca \& Bursztyn, 2009). 
A sociologia também entrou na discussão sobre inação, com destaque para os trabalhos de Eviatar Zerubavel. Seu argumento central é que a negação/inação não pode ser vista como fenômeno exclusivamente psicológico, mas que a seleção feita por indivíduos dos temas - que merecem atenção ou são ignorados - deve ser entendida dentro do contexto social atuante, que molda a interação interpessoal, assim como do contexto político. É o que o autor chama de organização social da negação, argumentando que se trata de um processo de resposta às circunstâncias sociais, desenvolvido pela interação social (Zerubavel, 1997; 2006; Norgaard, 2006).

A importância da teoria de Beck para as ciências sociais e para a compreensão de fenômenos naturais que apresentam ameaça às sociedades está no fato de ela considerar tais fenômenos como arenas de disputa política, exatamente porque os riscos estão "abertos a processos sociais de definição" (Beck, 2010: 27), e tudo o que for socialmente reconhecido como "causa" sofrerá pressões de mudanças. Como as relações de causalidade destes processos nem sempre são perceptíveis a priori, elas exigem uma explicação, podendo assim ser assimiladas e eventualmente aceitas como verdadeiras. Goldblatt (1996) refere-se a esse fenômeno como "invisibilidade social", o que diferencia os riscos ambientais de Beck das demais temáticas políticas. A ciência entra com o papel de trazê-los à tona, para que sejam contestados culturalmente.

Para Beck, a ausência das ciências sociais nas discussões sobre riscos ambientais deixa, muitas vezes, uma lacuna de extrema importância, sob o risco de "atrofiar-se numa discussão da natureza sem ser humano, sem questionar seu sentido social e cultural" (Beck, 2010: 29). Por ser relevante o conhecimento científico no reconhecimento público de alguns problemas, assim como em sua divulgação, a sociologia ambiental deve preocupar-se com as leituras culturais da natureza, ou com a forma como diferentes grupos sociais percebem e definem um problema ambiental (Lenzi, 2006). Beck (2009) revisitou posteriormente sua teoria da sociedade de risco no artigo "Critical theory of World Risk Society: a cosmopolitan vision". O autor apresenta então uma definição de risco muito útil para este trabalho:

Risk is not the same as catastrophe, but the anticipation of the future catastrophe in presence. As a result, risk leads a dubious, insidious, would-be, fictitious, allusive existence: it is existent and non-existent, present and absent, doubtful and real (Beck, 2009: 3).

A teoria da sociedade de risco apresenta um quadro alarmante, no qual a própria estrutura social - com suas normas, valores e tradições - é posta em risco pela emergência dos riscos ambientais modernos. É o que Merryn Ekberg (2007) chama de "onipresença do risco", quando um sentimento generalizado de vulnerabilidade toma a sociedade, ameaçando sua própria identidade. Tal condição é acompanhada pelos sentimentos de insegurança e incerteza que caracterizam o ethos da cultura de risco (Lash et alii, 
1996). A onipresença deste risco também é atribuída ao que Giddens (2002) chamou de "sequestro da experiência", devido à impossibilidade de se perceber certos riscos ambientais modernos, restando aos indivíduos confiar nas instituições especializadas, tendo suas vidas moldadas a partir de riscos imperceptíveis, reais ou não.

As mudanças climáticas são um caso eloquente que serve para fomentar essa discussão, já que um número crescente de pessoas testemunha possíveis provas destas transformações - incluindo a maioria dos entrevistados da pesquisa, como veremos mais adiante. Resta-nos ainda discutir não somente a relevância dessas percepções, mas também de que forma elas são moldadas por eventos climáticos perceptíveis, conectados ou não com o aquecimento global e por instituições que traçam as relações de causalidades mencionadas acima. Por exemplo, ao compararem percepções da população da Austrália e Grã-Bretanha, Reser et alii (2010) identificaram que, apesar dos níveis similares de preocupação, os australianos percebem o risco muito mais próximo: enquanto a porcentagem de pessoas que já estão sentindo os efeitos das mudanças climáticas é de 54\% (Austrália) e 41\% (Grã-Bretanha), os que esperam senti-los nos próximos $10-25$ anos é de $17 \%$ no primeiro caso e $27 \%$ no segundo. Eventos climáticos extremos locais e padrões climáticos variantes influenciam a percepção, aumentando a sensibilidade e localizando o risco.

Qual é então a utilidade de utilizar o construtivismo ambiental para se estudar este fenômeno? Analisar as mudanças climáticas a partir de uma abordagem construtivista não significa reduzir a existência do fenômeno natural à dimensão cognitiva dos seres humanos. As mudanças climáticas são um fenômeno físico, biológico, químico e geológico real, e não puramente uma construção social. Mas esta também é uma dimensão real do fenômeno, merecendo, portanto, o mesmo grau de atenção. A construção social das mudanças climáticas é induzida por uma organização social, ao mesmo tempo em que a influencia diretamente (Prades, 1999; Grundmann \& Stehr, 2010).

Estudar a realidade social da percepção de risco não significa abarcar toda a complexidade da experiência de insegurança ou medo, principalmente tratando-se dos riscos modernos apontados por Beck (2010). A experiência da percepção de risco não é algo uniforme, podendo assumir imagens confusas e contraditórias dentro de um mesmo grupo. Entretanto, exatamente por se tratar de uma teoria social, Beck assume que essa experiência pessoal e subjetiva é marcada por características culturais amplamente compartilhadas. Assim, assumir a percepção de risco como fato social é aceitar que sua distribuição pode ser, em maior ou menor escala, ordenada por características sociais fundamentais.

As teorias aqui analisadas permitem uma compreensão parcial da realidade cultural da percepção de risco, mas não são suficientes para abarcar toda a complexidade de 
como as pessoas percebem e reagem aos riscos diários. Ainda assim, compreender como uma sociedade percebe as mudanças climáticas como risco, quais as formas que essa construção social pode tomar e quais contextos afetam essa percepção é um caminho para compreender seu relacionamento com o ambiente que a cerca, com as instituições que com ela interagem, e mesmo com os meios de comunicação. É nesta justificativa teórica que este trabalho se insere e pretende contribuir, por meio da análise da percepção de risco no Brasil.

\section{Metodologia}

4. Os dados foram disponibilizados pela unidade responsável do Sesi-DN (Unitep). As informações sobre a metodologia da pesquisa foram retiradas do relatório do Ibope e adaptadas pelos autores. Todas as tabelas, gráficos, e análises deste artigo são de elaboração e responsabilidade própria dos autores. Convém ressaltar que não existem dados mais recentes e de livre acesso sobre o tema com a abrangência nacional. Apesar de pesquisas similares fornecerem ponto de comparação, como explorado mais à frente, as mesmas não possibilitariam o tratamento semelhante ao realizado neste trabalho.

5. A amostra foi elabora a partir de dados do Censo 2000, da Pesquisa Nacional por Amostra de Domicílios (Pnad) 2004 e do Tribunal Superior Eleitoral 2006.
Os dados utilizados neste artigo advêm da pesquisa de opinião pública sobre meio ambiente, realizada pelo Instituto Brasileiro de Opinião Pública e Estatística (Ibope, 2007) sob encomendada dos Departamentos Nacionais do Serviço Nacional de Aprendizagem Industrial (Senai-DN) e do Serviço Social da Indústria (Sesi-DN) ${ }^{4}$.

Os dados foram coletados entre os dias 13 e 18 de setembro de 2007, por meio de entrevistas pessoais, com utilização de questionários, realizadas por equipe de entrevistadores contratados pelo Ibope. O universo da pesquisa é composto por eleitores de 16 anos ou mais do Brasil. O universo foi estratificado por unidades da federação (UFs), com exceção dos estados do Acre, Amapá e Roraima, que juntos constituem um único estrato. Quando um estado possui região metropolitana, seu universo é novamente estratificado em "Região Metropolitana" e "Interior".

O modelo de amostragem utilizado é o de conglomerados, em três estágios. No primeiro, os municípios são selecionados probabilisticamente por meio do método PPT (Probabilidade Proporcional ao Tamanho) sistemático, com base na população de 16 anos ou mais de cada município ${ }^{5}$. No segundo estágio são selecionados os conglomerados (setores censitários) também por meio de PPT sistemático. A medida de tamanho é a população de 16 anos ou mais residente nos setores. Finalmente, no terceiro estágio, é selecionado, em cada conglomerado, um número fixo de pessoas segundo cotas de variáveis descritas abaixo:

$\checkmark$ Sexo: masculino e feminino.

- Grupos de idade: 16-17, 18-24, 25-29, 30-39, 40-49, 50-69 e 70 anos e mais.

- Instrução: apenas nível superior.

- Atividade: setor de dependência - agricultura, indústria de transformação, indústria de construção, outras indústrias, comércio, prestação de serviços, transporte e comunicação, atividade social, administração pública, outras atividades, estudantes e inativos. 
Essas cotas serviram para garantir a representatividade de grupos relevantes para a composição da amostra, mas não compõem sua totalidade ${ }^{6}$.

Foram realizadas 2.002 entrevistas em 142 municípios, o que possibilita um intervalo de confiança estimado de $95 \%$ e margem de erro máxima estimada de 2 pontos percentuais. Das entrevistas realizadas, retiraram-se as questões de interesse deste trabalho, as quais serão mencionadas na apresentação dos resultados a partir dos códigos apontados a seguir:

P1. Você diria que, os efeitos do aquecimento global:

a. já começaram a aparecer;

b. aparecerão nos próximos anos;

c. só aparecerão nas próximas gerações;

d. não sabe/não respondeu.

P2. Você acredita que o aquecimento global vai ou não vai afetar diretamente sua vida?

a. vai afetar;

b. não vai afetar;

c. não sabe/não respondeu.

P3. Em qual destas áreas você acredita que a sua vida será afetada?

a. saúde;

b. alimentação;

c. escassez de água;

d. falta de energia;

e. instabilidade no clima;

f. desemprego;

g. aumento de preços;

h. falta de peixes;

i. falta de dinheiro;

j. não sabe/não opinou.

P4. Pelo que você sabe ou ouve falar, para cada área citada:

P4A. aumento de doenças;

P4B. extinção de animais;

P4C. diminuição das vegetações;

P4D. acesso à água potável;

P4E. aumento da temperatura;

P4F. enchentes;

P4G. secas - diga o quanto o aquecimento global irá piorar cada uma

6. Para efeito de tabulação dos dados, foram consideradas as seguintes variáveis: sexo; grupo de idade $(16-24$ anos, 25-29, 30-39, 40-49, 50 anos e mais); escolaridade (até 4a série, 5a a 8a série, ensino médio, superior); renda familiar em SM (até 1, 1-2, 2-5, 5-10, mais de 10); região (Norte/Centro-Oeste Nordeste, Sudeste, Sul); condição do município (capital, periferia, interior); porte do município em número de habitantes (até 20 mil, 20-100 mil, mais de 100 mil). 
delas: "Muito ou pouco?"

a. muito;

b. pouco;

c. não irá piorar (explicitamente);

d. não sabe/não opinou.

P5. Com relação ao aquecimento global, você pessoalmente se considera:

a. muito preocupado;

b. preocupado;

c. pouco preocupado;

d. nada preocupado;

e. não sabe/não opinou.

\section{Resultados}

Os resultados aqui apresentados são divididos em duas partes principais. Em primeiro lugar, estão os resultados gerais da pesquisa, que dão suporte à hipótese de que a sociedade brasileira apresenta características da sociedade de risco de Beck. A percepção de risco analisada foi a respeito do aquecimento global e das mudanças climáticas, o que não significa que este seja o único responsável por essa construção social. Ao contrário, o caráter global da teoria da sociedade de risco leva a supor que o sentimento de insegurança ou medo diz respeito a uma diversidade de riscos ambientais que fazem parte da vida moderna, mesmo se analisado a partir de um risco específico.

A segunda parte diz respeito à distribuição social destes riscos, quando serão demonstrados cruzamentos entre as perguntas e características socioeconômicas, a fim de analisar quais variáveis são relevantes na distribuição da percepção de risco. Da mesma forma que na primeira parte, os resultados a serem apresentados podem servir como base para a compreensão da distribuição sociodemográfica de quaisquer riscos ambientais, respeitando as devidas particularidades.

\section{Dados gerais}

Ao desenvolver sua teoria, o foco dos trabalhos de Beck foram as sociedades modernas ocidentais que, tendo seus indivíduos cercados por ameaças ambientais desconhecidas, e a priori imperceptíveis, vivem numa atmosfera de insegurança generalizada. A assumida incapacidade tecnológica de controle das ameaças criadas pelo próprio avanço tecnológico faz com que nem mesmo as instituições modernas possam amenizar tal sentimento. Os dados analisados corroboram a ideia de que existe uma percepção de risco generalizada na população brasileira no que tange ao aquecimento global. Essa percepção é avaliada a partir de perguntas que não 
tocam diretamente a experiência do entrevistado: não se trata do risco a que ele/ ela está exposto/a, ou dos impactos que já sofreu. Apesar de poder ser contemplada, essa dimensão não é o foco da análise aqui proposta. Trata-se, por fim, de expectativas construídas a partir de informações recebidas, sejam elas experienciais ou não, corretas ou não: medo e insegurança quanto ao futuro, próximo ou distante, no que diz respeito ao aquecimento global e ao próprio bem-estar do indivíduo.

O aquecimento global já faz parte das preocupações da vida do brasileiro: somados, 85\% dos entrevistados acreditam que os efeitos do aquecimento global já começaram a aparecer, ou aparecerão nos próximos anos (Gráfico 1). O número de entrevistados explicitamente céticos quanto ao aparecimento desses efeitos é muito baixo

\section{GRÁFICO 1}

SOBRE OS EFEITOS DAS MUDANÇAS CLIMÁTICAS, SEU IMPACTO NA VIDA DO ENTREVISTADO E PREOCUPAÇÃO PESSOAL (QUESTÕES P1, P2 E P5)
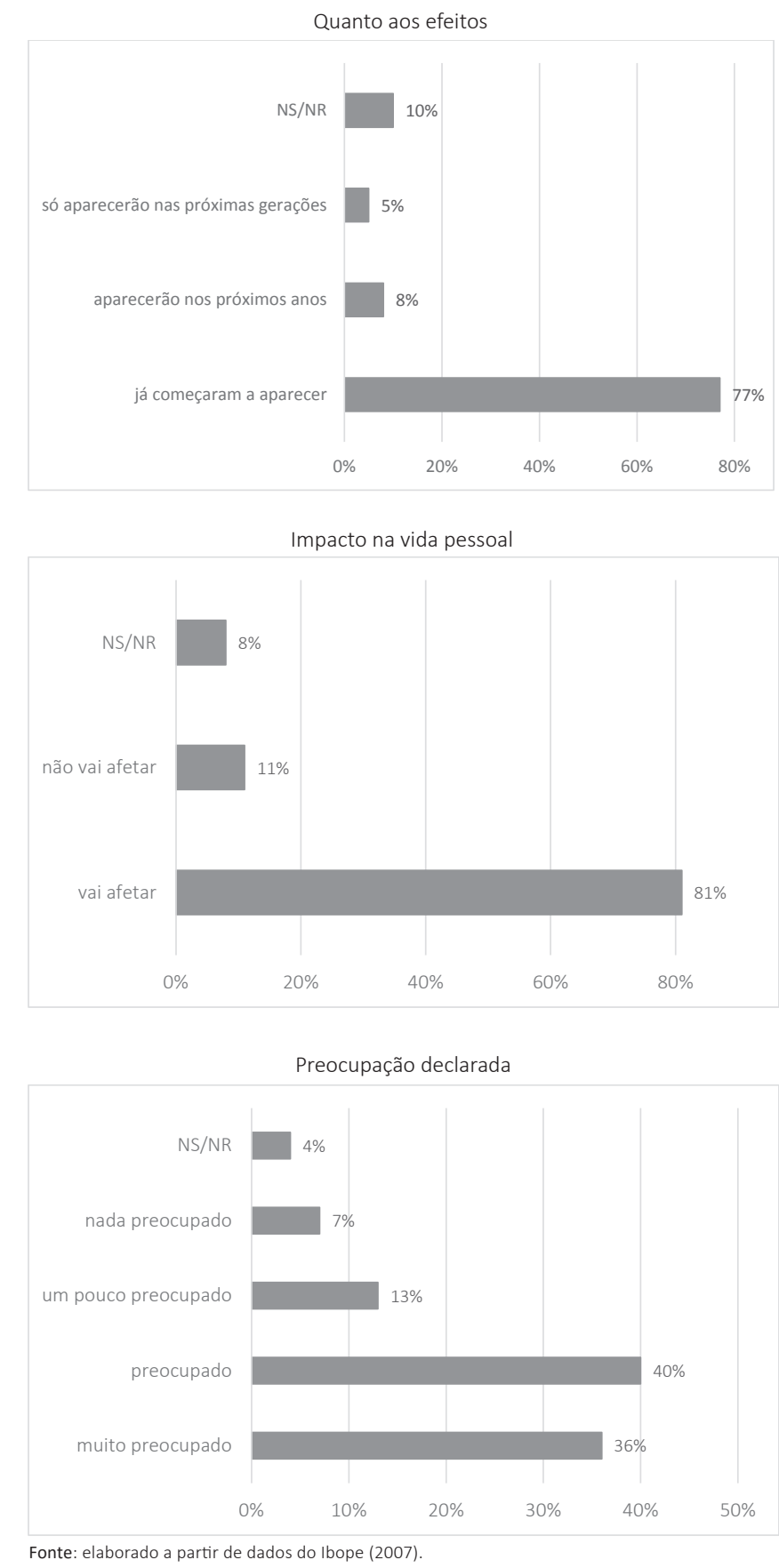
(5\%). Este resultado reflete a homogeneidade do acesso à informação sobre o tema, já que nem as estimativas mais pessimistas admitem uma percepção sensorial dos efeitos do aquecimento global em uma ordem tão alta (77\%).

A questão anterior tem relação direta com a preocupação dos indivíduos quanto às suas vidas: $81 \%$ dos entrevistados acreditam que sua vida será afetada diretamente pelo aquecimento global (Gráfico 1). Assim, podemos dizer que esta é a parcela da população brasileira que de alguma forma se preocupa com esse assunto, considerando-o como uma interferência real em suas vidas, no imediato ou no futuro próximo.

Quando questionados de forma mais direta sobre sua preocupação pessoal com o aquecimento global (Gráfico 1, Parte 3), 76\% dos entrevistados afirmaram estarem "preocupados" ou "muito preocupados", e apenas 6\% afirmaram estar "nada preocupados". Apesar de o valor agregado condizer com as demais questões, nota-se uma importante diferença na concentração de respostas na categoria mais pessimista, o que revela uma das características mais interessantes desse risco. Comparando as respostas mais pessimistas das três questões do Gráfico 1, tem-se: $77 \%$ dos entrevistados afirmaram que os efeitos do aquecimento global já começaram a aparecer; $81 \%$ acreditam que suas vidas serão afetadas por esse fenômeno; $77 \%$ acreditam que sua saúde será uma área afetada; e apenas 36\% se declararam "muito preocupados" com isso. O grupo foi divido, e pouco mais da metade (40\% do total) escolheu "preocupado" como a categoria que mais se identifica. Apesar da dificuldade de se interpretar exatamente o que é essa preocupação declarada e como ela se manifesta, esse aspecto dos dados não pode ser ignorado. Evitamos neste artigo comparar as categorias "preocupado" e "muito preocupado" em cada questão, por entendermos que em uma pesquisa de opinião pública a diferença empírica dessas respostas é de difícil interpretação. Vale ressaltar, porém, que essa foi a única questão em que a distribuição das respostas nessas categorias seguiu tal comportamento, o que pode ser interpretado como uma defasagem entre informação e preocupação, elementos que compõem a percepção de risco aqui analisada. Esta diferença pode ser apreciada pela negação mencionada por Zerubavel (2006), assim como pela influência de fontes de informação e o caráter generalista dos riscos modernos. Em outras palavras, o risco está presente na vida cotidiana e se trata de uma preocupação recorrente, ao mesmo tempo que é algo tão distante e incerto que, quando confrontado à vida concreta dos indivíduos, perde força.

A preocupação relatada acima é detalhada em algumas áreas da vida dos entrevistados (Gráfico 2, a seguir). "Saúde" e "escassez de água" são as áreas que os entrevistados, em sua grande maioria, se mostram mais preocupados (77\% e 69\% respectivamente). Quase a metade dos entrevistados se mostra apreensiva quanto à "alimentação", 
e um terço acredita que a "falta de energia" pode ser um problema. Por outro lado, áreas que dizem respeito a problemas econômicos ou de instabilidade no clima receberam valores irrisórios (de 0 a 2\% para todos os estratos). Isso leva à conclusão de que a preocupação dos entrevistados está diretamente vinculada a um risco físico, que ameaça sua saúde, bem como as necessidades básicas de água e alimentação.

Quando apresentados alguns problemas ambientais e questionados sobre o grau em que o aquecimento global pode piorar cada um deles, a categoria que mais recebeu a resposta "muito" foi o aumento da temperatura $(86 \%$ dos entrevistados, Gráfico 3).
GRÁFICO 2

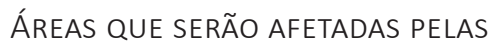
MUDANÇAS CLIMÁTICAS (QUESTÃO P3)

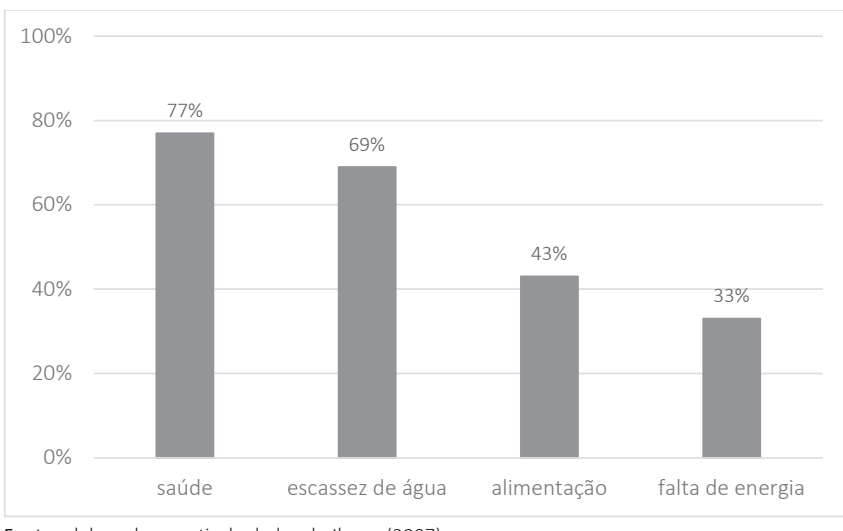

Fonte: elaborado a partir de dados do Ibope (2007).

\section{GRÁFICO 3}

EFEITO NEGATIVO DAS MUDANÇAS CLIMÁTICAS NAS ÁREAS MENCIONADAS (QUESTÃO P4)

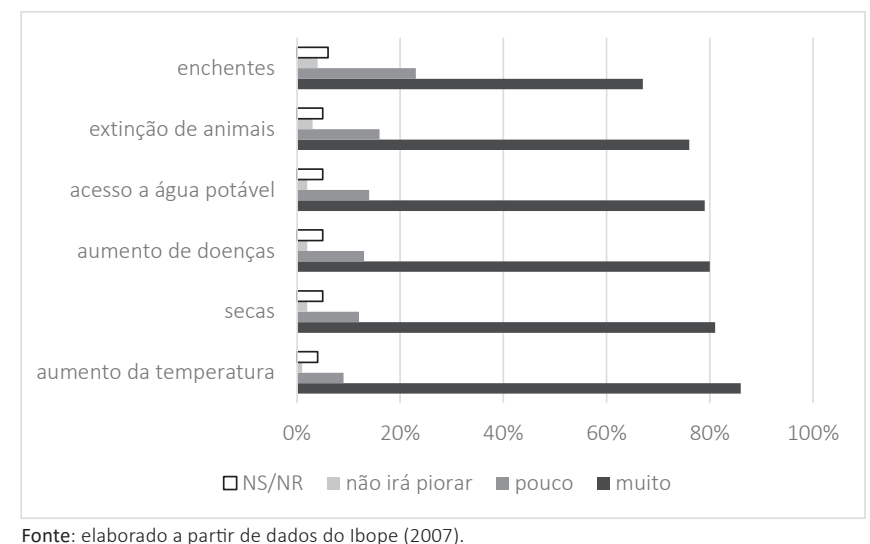

Fonte: elaborado a partir de dados do Ibope (2007).

Todos os demais problemas apresentados receberam um alto índice de respostas "muito", o menor sendo 67\% para "enchentes". A explicação para a homogeneidade das respostas para áreas tão distintas pode ser atribuída ao caráter generalista e apocalíptico das representações das mudanças climáticas por parte dos meios de comunicação em geral. Desta forma, para as pessoas que percebem a mudança do clima como um risco, todas as áreas citadas podem ser intensamente afetadas.

A falta de uma série histórica no Brasil não nos permite ainda chegar a conclusões sobre a dinâmica dessa percepção. 
É relevante assinalar que outro estudo realizado no mesmo ano (2007) aponta resultados similares: o Barômetro Ambiental, que conta com amostra composta por oito capitais brasileiras, chegou ao valor de $84 \%$ para aqueles acreditavam que os problemas ambientais afetariam seu cotidiano na próxima década, posicionando o Brasil em segundo lugar em uma comparação internacional entre 18 países, atrás apenas das Filipinas. Logo abaixo, estão Chile, Quênia, Índia e China - todos na ordem de 80\% - seguidos por Espanha, Canadá e Turquia (entre 74\% e 80\%) (Market Analysis, 2007).

Por meio da compilação de diversos trabalhos desenvolvidos nos Estados Unidos e na Europa, Spencer et alii (2010) apontam um pico na percepção de risco relacionada às mudanças climáticas, precisamente no período de coleta dos dados deste trabalho. Ao repetirem o estudo em 2010 na Grã-Bretanha, identificaram que 66\% dos entrevistados concordam que as mudanças climáticas representam um risco para as pessoas na Grã-Bretanha (eram 77\% em 2005) e 71\% estão preocupadas ( $82 \%$ em 2005), sendo que $78 \%$ consideram que o clima do mundo está mudando (91\% em 2005). No Brasil, apesar da diferença de formulação das questões, esse número permanece mais alto: $85 \%$ dos entrevistados acreditam que os efeitos do aquecimento global já começaram a aparecer, ou aparecerão nos próximos anos.

Ao comparar questões mais profundas, em estudo de percepções na Austrália e na Grã-Bretanha, observa-se a preocupação pessoal com as consequências das mudanças climáticas: 68\% na Austrália, 64\% na Grã-Bretanha (Reser et alii, 2010), e $81 \%$ no Brasil, como apresentado acima ("vida diretamente afetada"). Essas comparações ajudam-nos a ampliar a perspectiva dos resultados aqui apresentados, mas o principal, para este artigo, é qualificar esses resultados gerais, conforme apresentado a seguir.

\section{Distribuição social}

Nesta subseção é analisada a relevância de algumas variáveis na distribuição do risco associado às mudanças climáticas: escolaridade, região de domicílio, condição e tamanho do município e renda familiar. Das demais variáveis disponíveis, duas não estão aqui apresentadas. A variável "sexo" não apresentou qualquer interferência na estruturação das respostas, com uma diferença estatisticamente irrelevante (menos de $2 \%$ ) entre homens e mulheres no conjunto das respostas de opinião. Os testes feitos com a variável "idade" foram inconclusivos, apresentando comportamento irregular. Apesar disto, limitar uma conclusão sobre a relevância desta variável para a distribuição de risco, não dá indícios de comportamento estruturante, o que reforça a importância das demais variáveis apresentadas a seguir. 
A variável escolaridade apresentou grande influência em todas as perguntas. A tendência geral encontrada é que quanto maior a escolaridade, maior a percepção de risco a respeito das mudanças climáticas. $\mathrm{O}$ Gráfico 4 apresenta os indivíduos que deram respostas "preocupadas" em três diferentes perguntas, nas quais se observa a mesma tendência positiva. Para tal categorização, foram considerados os indivíduos que acreditam que os efeitos do aquecimento global "já começaram a aparecer" ou "aparecerão nos GRÁFICO 4 Respostas "Preocupadas" P1, P2 e P5 (POR ESCOLARIDADE)

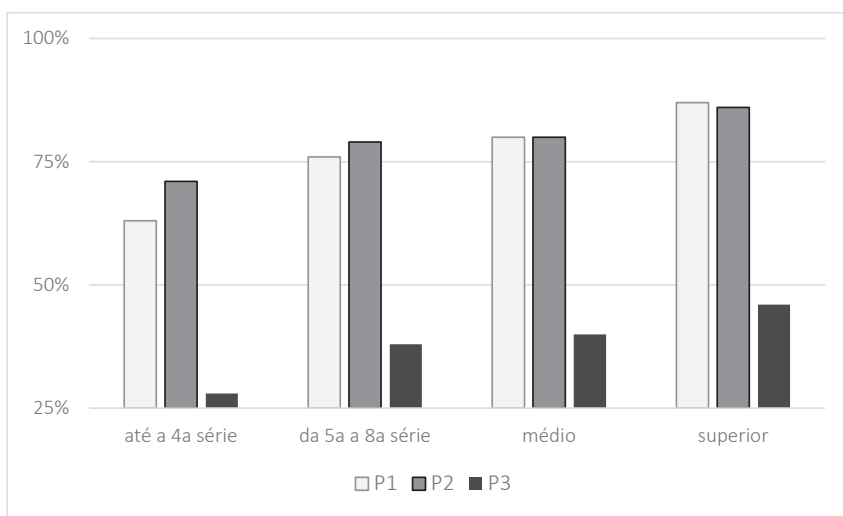

Fonte: elaborado a partir de dados do Ibope (2007). próximos anos" (P1); indivíduos que acreditam que terão suas vidas diretamente afetadas pelo aquecimento global (P2); e indivíduos que declaram estarem "preocupados" ou "muito preocupados" com o tema (P3).

Quando questionados sobre como o aquecimento global poderia piorar alguns problemas ambientais citados (questão P4), constata-se diferença significativa de respostas "muito" entre o primeiro e os últimos estratos, conforme está representado no Gráfico 5. Com uma exceção (enchentes), não existem grandes discrepâncias entre os entrevistados com ensi-

GRÁFICO 5

ÁREAS EM QUE O AQUECIMENTO GLOBAL TERÁ GRANDE EFEITO NEGATIVO (POR ESCOLARIDADE)

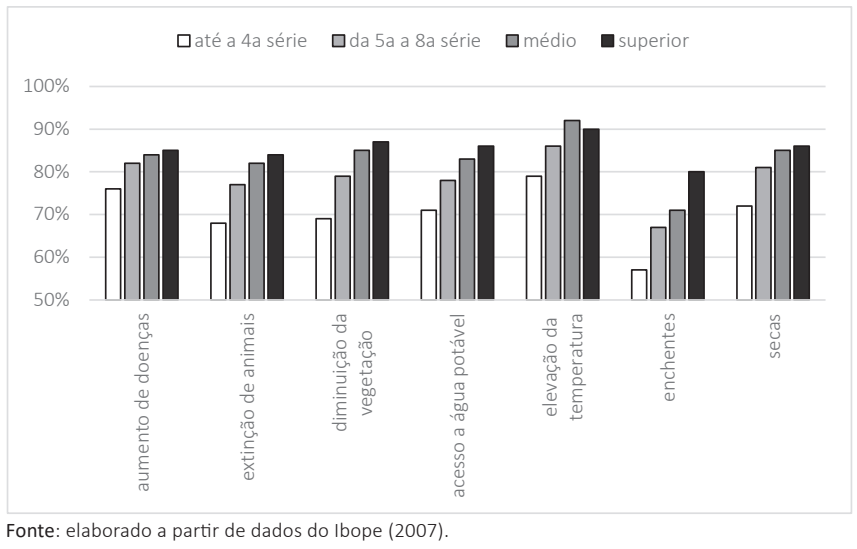


7. Essa aglomeração de não respostas também poderia indicar falta de compreensão por parte dos entrevistados por uso de linguagem inadequada. Apesar de admitir essa possibilidade, a solidez da estrutura de respostas em todas as questões permite manter as conclusões descritas. no médio e ensino superior, mas sim entre estes e aqueles dos estratos inferiores. A singularidade das respostas dos entrevistados com nível superior é sua convergência, sem grandes variações entre as áreas citadas (todas entre $80 \%$ e $90 \%$ ), diferentemente de todos os outros estratos.

Por fazerem pouca distinção entre os problemas mencionados, essa concentração relativa levanta a hipótese de que indivíduos com maior nível de escolaridade tendem a ver as mudanças climáticas como um risco global, tendo um efeito negativo generalizado. Para os entrevistados com menor nível de escolaridade, os problemas ambientais que mais serão agravados são os mais correntemente associados ao aquecimento global, como o aumento da temperatura e as secas. Na falta de teorias e estudos empíricos que comprovem essa relação, ressalta-se que se trata de hipótese a ser melhor testada, verificando se - e como - maior acesso a informação e maior grau de instrução interferem na compreensão desses fenômenos.

A incidência de não resposta é um importante termômetro para medir o grau de conhecimento e de interesse dos entrevistados no tema. Por isso a importância de se avaliar a percepção de risco, que passa necessariamente pelo acesso à informação. Ao confirmar a tendência acima já descrita, quanto menor a escolaridade, maior o índice de não resposta. Para os entrevistados de ensino médio e superior, todas as perguntas apresentam taxas abaixo de 5\%. Nos entrevistados com escolaridade até a 4⿳亠口冋 série, 5\% é o menor dos valores, encontrado em apenas uma pergunta (a que mais aborda opinião do entrevistado). A concentração das respostas está entre $10 \%$ e $15 \%{ }^{7}$.

Fica evidente, assim, que quanto maior a escolaridade, maior a percepção de risco. Outra conclusão geral é que a diferença entre entrevistados com nível médio completo e superior é pouca. A maior diferença se dá entre os níveis básicos de escolaridade. O "sequestro da experiência" de Giddens (2002) é aqui evidenciado como determinante na construção social do risco a partir do nível de escolaridade, acesso à informação e interpretação associada.

\section{REGIÃO GEOGRÁFICA}

A distribuição da percepção de risco por região apresentou poucas variações. A principal delas é a oposição entre os dois extremos formados pelas Regiões Nordeste e Sul. Grande parte dessa diferença se dá pela alta taxa de não resposta na Região Nordeste (Gráfico 6, a seguir).

Esta, porém, não é muito maior do que a taxa para a Região Sudeste e, visto a diferença de percepção de risco encontrada entre Nordeste e Sudeste, essa não pode ser a única explicação. Na primeira questão (P1), a diferença entre as Regiões Nordeste 
e Sul fica clara - $5 \%$ no Sul, $12 \%$ no Nordeste -, assim como é grande a diferença entre aqueles que acreditam que os efeitos do aquecimento global já começaram a aparecer (85\% no Sul, $69 \%$ no Nordeste), tendência que se repete na questão sobre a preocupação autodeclarada (P5).

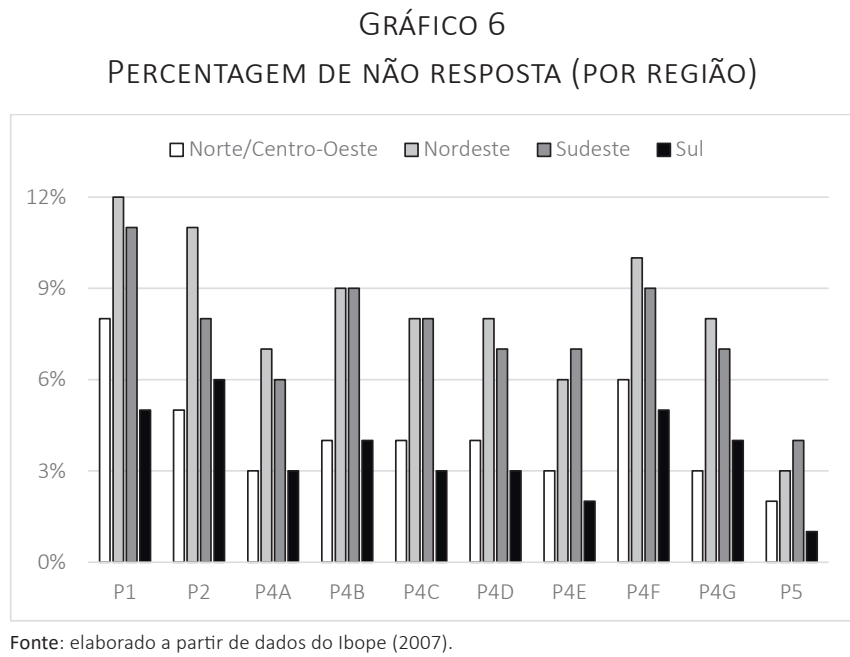

Fonte: elaborado a partir de dados do Ibope (2007)

Na questão sobre pro-

blemas ambientais que podem ser agravados com as mudanças climáticas (P4), existe uma diferença: a Região Nordeste continua apresentando menores valores de preocupação - neste caso, menos indivíduos afirmaram que o aquecimento global piorará "muito" em cada uma das áreas citadas. Porém, não é a Região Sul que apresenta os maiores valores, mas sim agrupamento Norte/Centro-Oeste. Quanto ao "aumento de temperatura", a área em que todas as regiões afirmaram que será a mais impactada, foi de 93\% para Norte/Centro-Oeste e $84 \%$ para o Nordeste. A categoria com menor nível de preocupação foi "enchentes", apresentando tendência diferente das demais, com maiores valores nas Regiões Sudeste (70\%) e Sul (69\%), números atribuídos ao fato das duas regiões estarem habituadas a esse tipo de catástrofe natural ${ }^{8}$.

As conclusões sobre a distribuição da percepção de risco por regiões do Brasil não são claras. Quando se trata de variáveis como escolaridade ou faixa etária, fica clara uma correlação entre acesso à informação e a construção de risco que permite explicar os comportamentos observados. No caso das regiões isso é um pouco mais difícil, mas a ausência de uma tendência estruturante também é relevante para a pesquisa, evidenciando mais uma vez o caráter homogeneizador da percepção de risco associada às mudanças climáticas.

\section{CONDIÇÃO DO MUNICÍPIO}

A variável "condição do município" está dividida em três categorias: capital, periferia (no caso de áreas metropolitanas) e interior. As variações encontradas nas diferentes questões não apontam uma tendência entre as categorias. Para ilustrar, na questão (P3) sobre as áreas da vida a serem afetadas, a diferença entre as quatro

8. Ressalta-se ainda que a pesquisa foi realizada antes das grandes enchentes do Nordeste em 2010, que afetaram principalmente os estados de Alagoas e Pernambuco. 
principais respostas não ultrapassa seis pontos percentuais. Para "saúde", ela é de 76\%, 77\% e 77\% para capital, periferia e interior respectivamente. Para "falta de energia", 29\%, 35\% e 35\%; "alimentação", 42\%, 48\% e 43\%; e "escassez de água", $72 \%, 72 \%$ e $67 \%$.

Em algumas questões, foi observada uma leve diminuição nos valores para a categoria "interior", mas muito pequena para permitir inferências analíticas. A explicação principal novamente reside na não resposta. Existe uma clara tendência de aumento desse tipo de resposta na direção capital-interior, com uma média de $4 \%$ para capitais, 5\% para periferia, e $8 \%$ interior. Mais uma vez, apesar de pequena em valores absolutos, a tendência não pode ser ignorada, por se repetir em todas as questões. Isso conduz a análise novamente à variável "nível de escolaridade", o que leva à conclusão de que "condição do município" não exerce influência sobre a distribuição de risco associado ao aquecimento global.

\section{TAMANHO DO MUNICÍPIO}

A variável "tamanho do município" foi dividida em três categorias: até 20 mil, de 20 a 100 mil e mais de 100 mil habitantes. Para a questão sobre os problemas ambientais a serem agravados (P4), a variação dos dados está abaixo de dez pontos percentuais para cada categoria, de $62 \%$ nos menores municípios a $71 \%$ nos maiores para "enchentes", e $83 \%$ a $86 \%$ para "aumento da temperatura". Isso indica que esta não é uma variável relevante na distribuição social da percepção de risco no Brasil. Mesmo se nos municípios com mais de 100 mil habitantes foi observada uma maior preocupação com relação aos efeitos negativos do aquecimento global, essa tendência acompanha o padrão de não resposta, que é levemente superior nos menores municípios.

Quando questionados sobre as áreas de suas vidas que seriam afetadas pelo aquecimento global (P3), as respostas não demonstram qualquer tendência, sem diferenças significativas de acordo com o tamanho do município. Por exemplo, para "saúde", as respostas são de 74\%, 75\% e 78\% (até 20 mil, de 20 a 100 mil e mais de 100 mil habitantes). Existe uma leve tendência de uma maior percepção de risco nos municípios com mais de 100 mil habitantes, orientação influenciada pelo alto índice de não resposta nos municípios menores. O comportamento mais disperso diz respeito à variável "falta de energia" (45\%, 29\% e 32\%, respectivamente), onde municípios pequenos apresentam maior preocupação, chegando a 16\% de diferença com municípios médios. Conclui-se, assim, que a variável "tamanho do município" não apresenta influência estruturante na percepção de risco, contando apenas com uma exceção à regra. 
A variável "renda familiar" foi dividida em cinco categorias, de acordo com o número de salários mínimos percebidos por todos os membros da família: mais de 10; de 5 a 10; de 2 a 5; de 1 a 2; até 1 . Nesta variável, a distribuição segue um claro padrão de que quanto maior a renda familiar, maior a percepção de risco. Com poucas exceções, a percentagem de entrevistados que afirmaram que o aquecimento global terá um

GRÁFICO 7

Problemas AMbientaIS QUE SERÃo AgRAVAdos PELO AQUECIMENTO GLOBAL (POR RENDA FAMILIAR) impacto "muito" negativo nas categorias citadas na questão P4 cai progressivamente com a queda da renda familiar, como mostra o Gráfico 7.

Essa tendência fica mais perceptível quando o entrevistado é questionado sobre sua preo-

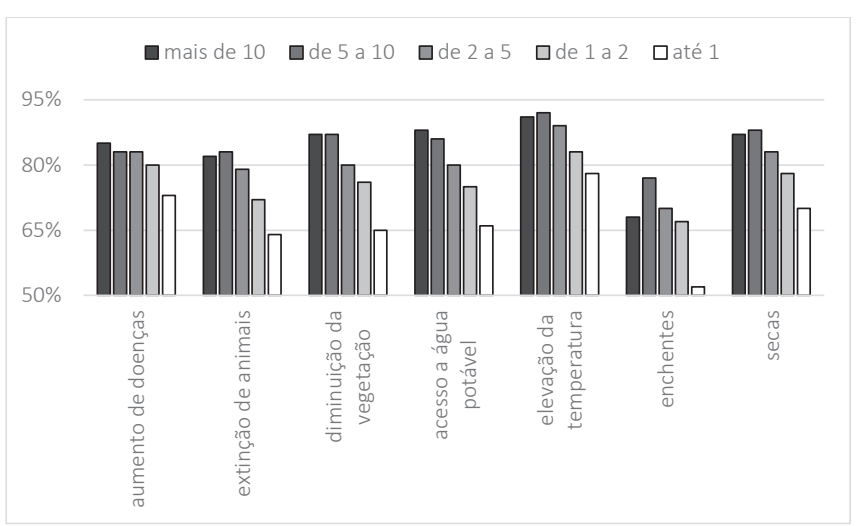
Fonte: elaborado a partir de dados do Ibope (2007). tema (P5). A diferença entre os grupos extremos é evidente: do grupo de maior renda, nenhum entrevistado se declarou "nada preocupado" com o aquecimento global, contra $15 \%$ do grupo até 1 salário mínimo. Da mesma forma, somando as categorias "preocupados" e "muito preocupados", encontramos no estrato "mais de 10 salários mínimos" um total de $88 \%$ dos entrevistados, enquanto esse número cai para $63 \%$ no outro extremo dos entrevistados.

Também se constatou uma grande diferença entre aqueles que afirmam que os efeitos do aquecimento global já começaram a aparecer (P1): 93\% entre os que ganham mais de 10 SM e 58\% no grupo de até 1 SM. É importante notar também que a categoria "não sabe/não respondeu" aumenta sistematicamente à medida que a renda diminui, indo de 1\% a 21\% (maior valor de não resposta em todos os cruzamentos feitos). Mais uma vez, as não respostas aparecem como um dado importante na interpretação dos resultados.

Como outras variáveis analisadas neste artigo, renda familiar aparece associada ao nível de escolaridade. Com uma correlação ainda mais intensa, esta variável é ainda mais relevante na distribuição da percepção de risco. Desta forma, a solidez dos 
dados observados permite concluir que a distribuição da percepção de risco nos estratos econômicos da sociedade brasileira é bem definida: quanto maior a renda, mais o indivíduo conhece e se preocupa com o aquecimento global.

Existe uma tendência em trabalhos sobre a esfera social dos efeitos das mudanças climáticas, de que os estratos mais pobres das sociedades são mais vulneráveis a desastres ambientais que podem ser agravados pelas mudanças climáticas, seja como resultado de mal planejamento urbano, ou acesso a tecnologias de adaptação no campo (Adger, 2003; Smit \& Wandel, 2006; Hardoy \& Pandiella, 2009; IPCC, 2001; 2007). Por outro lado, a importância dos dados aqui analisados está em mostrar que o outro extremo da pirâmide socioeconômica da sociedade brasileira tampouco se sente imune a esses riscos. Não se pode tirar conclusões sobre o medo relativo das camadas da sociedade e entender como cada uma delas se sente vulnerável aos riscos em comparação com as demais. O alto índice de não resposta nos estratos mais baixos não permite esse tipo de comparação mais direta, pois, apesar de compor a percepção de risco, esconde outros fatores relevantes como a não compreensão das questões. Ainda assim, o fato de fazer parte do estrato econômico mais alto da sociedade não tira dos indivíduos a sensação de insegurança ou incerteza associado ao fenômeno.

\section{Considerações finais}

Existe uma relevante uniformidade na percepção de risco das mudanças climáticas da população brasileira, onde a grande maioria dos entrevistados acredita que os efeitos do aquecimento global já começaram a aparecer ou aparecerão nos próximos anos, e que suas vidas serão afetadas diretamente por eles.

Esta uniformidade pode ser interpretada a partir da teoria de Beck - e contribuições de Giddens - quanto à natureza imperceptível dos riscos modernos, restando aos indivíduos confiar nas instituições especialistas, e também nos meios de comunicação que transmitem tal conhecimento.

Levanta-se a hipótese de que o fator responsável pela homogeneidade da percepção de risco encontrada na pesquisa é a televisão, que continua a ser o canal de informação mais importante no Brasil em todas as camadas sociais, presente em 96,88\% dos domicílios - mesmo na Região Norte, com menor presença da televisão, essa taxa chega a 91,66\% (IBGE, 2011). A relevância desta hipótese é reforçada por Eiró e Lindoso (2014) em estudo sobre a percepção de risco de agricultores familiares do semiárido brasileiro. Mais da metade de seus entrevistados (em amostra de quatro municípios na Bahia) já tinha ouvido falar dos termos "mudanças climáticas" e "aquecimento global", dos quais $90 \%$ mencionaram a televisão como a fonte, e em 
segundo lugar o rádio, com 26\%. Por fim, existem indícios de aumento da cobertura sobre o tema na mídia. Tomando o exemplo de um importante jornal brasileiro, $A$ Folha de S. Paulo, no ano de realização da coleta de dados deste trabalho (2007), o número de artigos com menções combinadas dos termos "mudanças climáticas", "efeito estufa" e "aquecimento global" foi três vezes superior que sua média dos dez anos anteriores (Market Analysis, 2007).

Apesar de a pesquisa não ter tratado esse aspecto, a fraca interferência de variáveis demográficas (sexo, idade, região, tamanho e condição do município) na distribuição do risco permite tal constatação. Apenas duas das variáveis analisadas se mostraram estruturantes: renda familiar e escolaridade. Ambas com o mesmo comportamento positivo: quanto maior a renda familiar ou a escolaridade, maior a percepção de risco. É preciso mencionar que existe uma ligação direta entre as duas variáveis no caso brasileiro, e que o acesso à informação e a sua interpretação por pessoas com maior escolaridade explica essa estratificação de respostas.

Quanto às demais variáveis, algumas categorias de respostas demonstraram comportamentos específicos, mas nenhum efeito estrutural corroborado em todas as questões. Já variáveis geográficas (região, tamanho do município, e condição do município) não apresentaram qualquer tendência, indicando sua fraca ou ausente influência sobre a percepção de risco e a necessidade de estudos mais aprofundados.

Ao retomar o tema da natureza deste risco, chega-se a duas outras conclusões a partir dos dados analisados. A primeira delas é que se trata de uma percepção de risco generalizada, na qual todas as áreas da vida podem ser afetadas. Essa característica é identificada como "onipresença do risco", ou o sentimento geral de vulnerabilidade da sociedade de risco (Ekberg, 2007). A segunda característica diz respeito ao risco físico das mudanças climáticas. Existe um consenso (onde nenhuma variável sociodemográfica apresentou efeito relevante) quanto aos aspectos da vida em que os entrevistados mais temem impactos negativos da mudança do clima, notadamente "saúde" e "escassez de água e alimentos".

Finalmente, duas discussões merecem ser levantadas, como questionamentos relevantes para o tema, que derivam das análises realizadas. A primeira delas diz respeito à ideia de que indivíduos inseridos em diferentes contextos sociais e com diferentes experiências de vida interpretam riscos de maneiras diferentes. Apesar de não se levantar aqui dúvidas sobre essa hipótese - especialmente por abranger profundos níveis de subjetividade humana -, é preciso relativizar tal contextualização ao se tratar de percepção de risco. Como já mencionado, a natureza "imperceptível" dos riscos modernos aqui tratados diminui a interferência de contextos individuais na construção dos mesmos. Ou dito de outra maneira, o significado que cada indivíduo 
atribui às informações que recebe divergem em níveis muito subjetivos, que parecem ter pouca influência na concepção geral que se tem dos riscos, e nas formas de reagirem a eles.

Litre et alii (2014) encontraram resultados semelhantes no que toca à homogeneidade da percepção de risco de agricultores familiares de três biomas brasileiros - Amazônia, Cerrado e Caatinga. Mesmo inseridos em ambientes completamente distintos e desenvolvendo atividades sensíveis a variações climáticas, tal percepção convergente também estava relacionada com estratégias de adaptação, o que nos leva à segunda discussão que complementa o ponto anterior e se resume na pergunta: qual o grau de coesão entre o discurso e o medo real experimentado pelos indivíduos no que tange o aquecimento global?

Em um contexto distinto, Norgaard (2006) identificou uma série de estratégias utilizadas por comunidades rurais na Noruega para evitar o assunto "mudanças climáticas". Mesmo tendo informações suficientes sobre o tema, discuti-lo significaria evocar emoções indesejadas de medo, o que resulta em uma negação coletiva e consequente inação. Essa incoerência entre percepção e ação deve ser tomada em conta para não conduzir a uma interpretação precipitada de generalização de um sentimento constante de insegurança. Apesar de não ser o foco deste trabalho, a relação entre percepção de risco e atitudes de mitigação dos efeitos do aquecimento global, ou de proteção a eles, seria uma forma de se avaliar o medo real. Esses são questionamentos possíveis, que podem ser objeto de pesquisas futuras para se aprofundar a compreensão da formação da percepção de risco e seus desdobramentos.

Abstract: This article aims to test the applicability of the Risk Society Theory proposed by Ulrich Beck for the contemporary Brazilian society, and to analyze its social distribution of risk perception associated with climate change and global warming. To do so, it reviews risk theories and discusses critically its application to the case of climate change. It presents then the results of the poll, which checked the effect of different socio-demographic variables on risk perception. The data come from public opinion poll in national scale, with stratified cluster sample. The main result achieved regards the homogeneity of risk perception across different social categories or geographical contexts. The only socio-demographic categories that showed significant influences on the assessment of risk perception were household income and education, both with a positive relationship.

Keywords: climate change, global warming, risk, perception, constructivism. 


\section{Referências}

ADGER, W. N. Capital, Collective action, and adaptation to climate change. Economic Geography, v. 79, n. 4, p. 387-404, 2003.

BECK, U. Sociedade de risco: rumo a uma outra modernidade. São Paulo: Editora 34, 2010 [1992].

. Critical theory of world risk society: a cosmopolitan vision. Constellations, v. 16, Issue 1, p. 3-22, 2009.

- World risk society. Malden (MA): Polity Press, 1999.

_. Ecological politics in an age of risk. Cambridge: Polity Press, 1995.

BÉRUBÉ, C. Changements climatiques et distorsion de la perception des québécois: de la communication à l'action. Essai de maître en environnement, Université de Sherbrooke, 2010.

DOUGLAS, M. Risk and blame: essays in cultural theory. London: Routledge, 1994.

EIRÓ, F. Mudanças climáticas e percepção de risco na Amazônia e no Semiárido brasileiros. Dissertação de mestrado, Universidade de Brasília, 2012.

EIRÓ, F.; LINDOSO, D. Mudança climática, percepção de risco e inação no Semiárido brasileiro: como produtores rurais familiares percebem a variabilidade climática no Sertão do São Francisco - Bahia. Revista Econômica do Nordeste, v. 45, n. 4, 2014.

EKBERG, M. The parameters of the Risk Society: a review and exploration. Current Sociology, v. 55, n. 3, p. 343-366, 2007.

FONSECA, I. F.; BURSZTYN, M. A banalização da sustentabilidade: reflexões sobre governança ambiental em escala local. Sociedade e Estado, v. 24, n. 1, p. 17-46, 2009.

GIDDENS, A. Modernidade e identidade. Rio de Janeiro: Editora Jorge Zahar, 2002.

GOLDBLATT, D. Social theory and the environment. Cambridge: Polity Press, 1996.

GRUNDMANN, R.; STEHR, N. Climate change: what role for sociology?: A response to Constance Lever-Tracy. Current Sociology, v. 58, n. 6, p. 897-910, 2010.

HABERMAS, J. The theory of communicative action. Boston: Beacon Press, 1984.

HARDOY, J.; PANDIELLA, G. Urban poverty and vulnerability to climate change in Latin America. Environment and Urbanization, n. 21, p. 203-224, 2009. 
INSTITUTO BRASILEIRO DE GEOGRAFIA E ESTATÍ́sTICA (IBGE). Pesquisa Nacional por Amostra de Domicílios. Tabela 1154, 2011. Disponível em: <http://www.sidra. ibge.gov.br/>. Acesso em: 17 Abr. 2013.

INSTITUTO BRASILEIRO DE OPINIÃO PÚBLICA E ESTATÍ́sTICA (IBOPE). Pesquisa de opinião pública sobre o aquecimento global. Relatório de pesquisa. Brasília: Sesi-DN; Senai-DN, 2007.

INTERGOVERNMENTAL PANEL ON CLIMATE CHANGE (IPCC). Climate Change 2007: synthesis report. Contribution of Working Groups I, II, III to the Fourth Assessment Report of the Intergovernmental Panel on Climate Change. Geneva: WMO, IPCC Secretariat, 2007.

_. Climate change: impacts, adaptation and vulnerability. Valência: IPCC, 2001.

JOFFE, H. Risk: from perception to social representation. The British Journal of Social Psychology, v. 42, p. 55-73, 2003.

LASH, S.; SZERSZYNSKI, B.; WYNNE, B. Risk, environment and modernity: towards a new ecology. London: Sage Publications, 1996.

LENZI, C. Sociologia ambiental: risco e sustentabilidade na modernidade. Bauru: EdUSC, 2006.

LEVER-TRACY, C. Global warming and sociology. Current Sociology, v. 56, n. 3, p. 445-466, 2008.

LIDSKOG, R. The re-naturalization of society? Environmental Challenges for Sociology. Current Sociology, v. 49, n. 1, p. 113-136, 2001.

LITRE, G; NASUTI, S.; GUCCIARDI GARCEZ, C. A.; LINDOSO, D. P.; EIRÓ, F.; SIMONI, J.; SILVA, C. J.; FAÇANHA, C. L. From rainforests to drylands: comparing family farmers perceptions of climate change in three brazilian biomes. In LEAL FILHO, W. ; ALVES, F.; CAEIRO, S.; AZEITEIRO, U. M. (Orgs.). Climate change management, p. 165-185. Springer International Publishing, 2014.

MARKET ANALYSIS. Rumo a um novo consenso pela sustentabilidade? Os brasileiros diante das mudanças climáticas. Florianópolis: Market Analysis Brasil, 2007.

MORA, M. La teoría de las representaciones sociales de Serge Moscovici. Athenea Digital, v. 2, 2002.

MOSCOVICI, S. Representações sociais: investigações em psicologia social. Petrópolis: Vozes, 2003. 
NORGAARD, K. People want to protect themselves a little bit: emotions, denial, and social movement nonparticipation. Sociological Inquiry, v. 76, n. 3, p. 372-396, 2006.

OLIVIERI, A. G. A teoria da modernização ecológica: uma avaliação crítica dos fundamentos teóricos. Tese de Doutorado em Sociologia, Universidade de Brasília, 2009.

OLSON, M. A lógica da ação coletiva: os benefícios públicos e uma teoria dos grupos sociais. São Paulo: EdUSP, 1999.

PRADES, J. A. Global environmental change and contemporary society: classical sociological analysis revisited. International Sociology, v. 14, n. 1, p. 7-31, 1999.

SANCOVSCHI, B. Sobre a noção de representação em S. Moscovici e F. Varela. Psicologia e Sociedade, v. 19, n. 2, p. 7-14, 2007.

SMITH, M. The face of nature: environmental ethics and the boundaries of contemporary social theory. Current Sociology, v. 49, n. 1, p. 49-65, 2001.

SMIT, B.; WANDEL, J. Adaptation, adaptive capacity and vulnerability. Global Environmental Change, v. 16, p. 282-292, 2006.

SPENCER, A.; VENABLES, D.; PIDGEON, N.; POORTINGA, W.; DEMSKI, C. Public perceptions of climate change and energy futures in Britain: Summary findings of a survey conducted in january-march 2010. Technical Report, Understanding Risk Working Paper, 10-01. Cardiff: School of Psychology, 2010.

RESER, J. P.; PIDGEON, N.; SPENCE, A.; BRADLEY, G.; GLENDON, A. I.; ELLUL, M. (2010) Public risk perceptions, understandings, and responses to climate change in Australia and Great Britain: interim report. Cardiff University, Wales: Griffith University, Climate Change Response Program, Queensland, Australia, and Understanding Risk Centre, 2010.

WILKINSON, I. Social theories of risk perception: at once indispensable and insufficient. Current Sociology, v. 49, n. 1, p. 1-22, 2001.

ZERUBAVEL, E. The elephant in the room: silence and denial in everyday life. Oxford: Oxford University Press, 2006.

- Social mindscapes: an invitation to cognitive sociology. Cambridge (MA): Harvard University Press, 1997. 
